\title{
Role of Molecular Complexation in Solid-Liquid Phase Diagram of Poly- ethylene Oxide/Urea Mixture
}

\author{
Guopeng Fu and Thein Kyu* \\ Department of Polymer Engineering, University of Akron, OH 44325, USA. \\ Email: thyu@uakron.edu \\ Telephone: +1 (330) 9726672
}

\begin{abstract}
Thermodynamic phase diagram of polyethylene oxide (PEO)/urea blend has been investigated with emphasis on the formation of induced crystals driven by molecular complexation. Of particular interest is that the complexed $\alpha$ crystal is more stable than those of neat constituents. The experimental solid-liquid phase diagram of PEO/urea blends has been analyzed in the context of the combined Flory-Huggins free energy for liquid-liquid demixing and phase field free energy of crystal solidification. The self-consistently calculated coexistence lines of the PEO/urea mixture exhibit an azeotrope type, accompanied by eutectoid reactions in the submerged regions, wherein the complexed $\alpha$ crystal decomposes into two coexistence crystalline phases. These coexistence crystalline phases were further analyzed by means of polarized optical microscopy, Fourier transform infrared spectroscopy, and wide-angle x-ray diffraction. A possible phase diagram of the complexed PEO/urea is constructed and discussed.
\end{abstract}

(C) 2017. This manuscript version is made available under the Elsevier user license http://www.elsevier.com/open-access/userlicense/1.0/ 
Introduction

Molecular complexation between the small-molecule hosts and linear polymer guests has gained considerable interest because of the formation of inclusion crystal compounds.[1-11] Typical examples include urea (or thiourea) hosts and polyolefinic guest macromolecules, where the host urea forms a network of crystalline channels driven by intramolecular or self-hydrogen bonding as the non-polar guest macromolecules weave through the urea crystalline channels. Such inclusion compound was reported to be stabilized by the van der Waals forces between the polar host and non-polar guest molecules.[10,11] In the case of polar guest molecules such as polyethylene oxide (PEO), their mixtures with urea host were found to form a stable inclusion crystalline compound via inter-species hydrogen bonding, termed as the $\alpha$ crystals. $[12,13]$ By virtue of inter-species hydrogen bonding between the constituent polar molecules, the melting temperatures of the $\alpha$ crystals in the intermediate PEO/urea blend compositions were found to be considerably higher than those of the neat constituents.[14, 15] Upon lowering temperature, there appeared another compound called $\beta$ crystal in the PEO-rich region that melts in the vicinity of $\sim 100{ }^{\circ} \mathrm{C}$, i.e., intermediate between those of neat PEO crystal (i.e., $65{ }^{\circ} \mathrm{C}$ ) and of the $\alpha$ crystal (i.e., $144{ }^{\circ} \mathrm{C}$ ).[16-18] The $\beta$ crystal was characterized as a metastable crystal that transformed reversibly to the stable $\alpha$ crystal upon heating and cooling.[16] However, this $\beta$ crystal was absent in a similar work[19] on PEO (a lower molecular weight)/urea blends, and thus these findings were at odd to each other.

In this paper, we attempt to explain the binary crystalline phase diagram of PEO/urea mixture in the framework of the combined Flory-Huggins free energy for liquid-liquid demixing and the Landau-type phase field free energy of crystal solidification.[20-24] The self-consistent solution to the combined free energy description reveals that the observed phase diagram is of an azeotrope type forming complexed $\alpha$ crystal + isotropic liquid coexistence regions. Upon lowering temperature, this $\alpha$ crystal undergoes a eutectoid reaction that further decomposes into two separate crystalline coexistence regions, viz., (urea crystal $+\alpha$ crystal) and ( $\alpha$ crystal $+\beta$ crystal) regions. A possible phase diagram is constructed by comparing the self-consistently calculated curves versus experimentally determined coexistence regions.

\section{Model description}

The total free energy density of a binary crystalline polymer blend may be expressed as a sum of the free energy of Flory-Huggins theory (FH) for liquid-liquid demixing and the phase field (PF) free energy for crystal solidification,[20-24] and their coupling term involving anisotropic interaction parameters representing crystallineamorphous $\left(\chi_{c a}\right)$, amorphous-crystalline $\left(\chi_{a c}\right)$ and crystalline-crystalline $\left(\chi_{c c}\right)$ interaction; $[23,24]$

$$
\begin{aligned}
f\left(\psi_{1}, \psi_{2}, \phi_{1}, \phi_{2}\right) & =\phi_{1} f\left(\psi_{1}\right)+\phi_{2} f\left(\psi_{2}\right)+\left(\frac{\phi_{1}}{r_{1}} \ln \phi_{1}+\frac{\phi_{2}}{r_{2}} \ln \phi_{2}+\chi_{a a} \phi_{1} \phi_{2}\right) \\
& +\left(\chi_{c a}{\psi_{1}}^{2}-2 \chi_{c c} \psi_{1} \psi_{2}+\chi_{a c}{\psi_{2}}^{2}\right) \phi_{1} \phi_{2}(1)
\end{aligned}
$$

$\psi_{1}$ and $\psi_{2}$ represent the linear crystallinity of component 1 and 2 , respectively. The first and second terms represent the Landau-type phase field free energy of crystal solidification of each constituent, $f\left(\psi_{1}\right)$ and $f\left(\psi_{2}\right)$, in which the individual free energy of the constituents is coupled to their respective volume fractions. The third term represents the Flory-Huggins (FH) free energy for liquid-liquid demixing, where $\phi_{1}$ and $\phi_{2}$ are the volume fractions of the 
component 1 and component 2, respectively, satisfying the incompressibility condition, i.e., $\phi_{1}+\phi_{2}=1$. The parameters $r_{1}$ and $r_{2}$ represent the numbers of statistical segments of each component. Flory-Huggins interaction parameter, $\chi_{a a}$ is taken as $\chi_{a a}=A+B / T$, representing amorphous-amorphous interaction, where $\mathrm{A}$ and $\mathrm{B}$ are constants.

The free energy of crystallization may be described in the framework of phase field theory using a Landau-type asymmetric free energy.[23] We recently extended the phase field theory to elucidate crystallization behavior of a binary crystalline polymer blends,

$$
f_{\text {local }}\left(\psi_{i}\right)=W_{i}\left[\zeta_{i} \zeta_{0, i} \frac{\psi_{i}^{2}}{2}-\left(\zeta_{i}+\zeta_{0, i}\right) \frac{\psi_{i}{ }^{3}}{3}+\frac{\psi_{i}^{4}}{4}\right]
$$

where $\zeta_{i}$ is the crystalline order parameter at the stable potential well for the crystal solidification in which subscript $i$ represents component 1 or $2 . \zeta_{0, i}$ is the crystalline order parameter representing unstable energy barrier for nucleation to overcome. The coefficient $W_{i}$ is related to the nucleation process to overcome the energy barrier. $\chi_{c a}=\chi_{c 1 a 2}$ represents the anisotropic interaction parameter between the crystalline solid of the first constituent and the amorphous liquid of the second, and vice versa $\chi_{a c} \cdot \chi_{c c}$ is the crystalline-crystalline interaction parameter, which may be expressed in terms of geometric means of the crystal-amorphous and amorphous-crystal interactions. To account for non-ideality, Matkar and Kyu[23] proposed that

$$
\chi_{c c}=c_{\omega} \sqrt{\chi_{c a} \cdot \chi_{a c}}
$$

where $c_{\omega}$ is the anisotropic interaction strength. When $c_{\omega}<1$, the crystalline system is destabilized and forms separate crystals, while exhibiting a eutectic or peritectic behavior. On the other hand, when $\mathrm{c}_{\omega}>1$, the crystallinecrystalline interaction is stronger than the crystal-amorphous and/or amorphous-crystal interactions, and thus the system becomes more stable in the mixture that forms an azeotrope-type co-crystal. The ideal case occurs when $\mathrm{c}_{\omega}=$ 1 , wherein the crystal melting temperatures vary linearly with composition. A narrow loop develops if $\mathrm{c}_{\omega}=1$. By varying the $\mathrm{c}_{\omega}$ parameter, Matkar and Kyu demonstrated that the aforementioned combined FH/PF theory can capture various phase diagrams encompassing azeotrope, ideal loop, peritectic to eutectic behaviors of binary crystalline polymer blends.[23]

\section{Materials and Methods}

Materials and sample preparation

PEO powders having a weight-average molecular weight of 35,000 g/mole and urea with a molecular weight of 60 $\mathrm{g} /$ mole were purchased from Aldrich Chemical Co. Various compositions of the PEO/urea blend were prepared by dissolving in methanol (Fisher Scientific, Inc.) individually to form a $5 \mathrm{wt} \%$ polymer solution and subsequently mixed them for $2 \mathrm{~h}$ at $50{ }^{\circ} \mathrm{C}$ to ensure complete dissolution of both constituents. Some solution cast samples were further heated to $150{ }^{\circ} \mathrm{C}$ in vacuum oven, then the samples were cooled down to room temperature, hereafter denoted as thermally annealed blends. 
Methods

Thermal transition behaviors of various PEO/urea blends were examined by means of differential scanning calorimetry (DSC, Model 2920 Thermal Analysis Instruments, TA) at various heating rates of 2, 5, and $10{ }^{\circ} \mathrm{C} / \mathrm{min}$, but only the $2{ }^{\circ} \mathrm{C} / \mathrm{min}$ scans were used in the phase diagram determination because the melting temperatures thus obtained at $2{ }^{\circ} \mathrm{C} / \mathrm{min}$ were relatively closer to the equilibrium temperature. Infrared spectra of all $\mathrm{PEO} /$ urea blends were acquired using a Fourier transform infrared (FTIR) spectrometer (Nicolet 380) to examine molecular interactions between amine group of urea and ether group of PEO. The film samples (50 $\mu \mathrm{m}$ thick) for FTIR were solution-cast on $\mathrm{KBr}$ discs from the $5 \mathrm{wt} \%$ polymer solution in methanol and dried under vacuum at room temperature for $24 \mathrm{~h}$ to remove residual solvent. The acquired IR spectra represented the average of 64 scans with a resolution of $2 \mathrm{~cm}^{-1}$. The development of phase morphology was examined on an optical microscope (BX60, Olympus) using a sample hot stage and a temperature controller (TMS 93, Linkam). The phase morphologies were photographed using a $35-\mathrm{mm}$ digital camera (EOS 400D, Canon) at 50× magnification unless indicated otherwise. Various samples with different compositions were further analyzed by means of wide-angle X-ray diffraction (WAXD) using a sealed tube X-ray generator (Bruker Instrument) with the monochromatized $\mathrm{Cu}-\mathrm{K}_{\alpha}$ radiation having a wavelength of $1.5417 \AA$. The pinhole collimator having $0.5 \mathrm{~mm}$ in diameter was used.

Results and Discussion

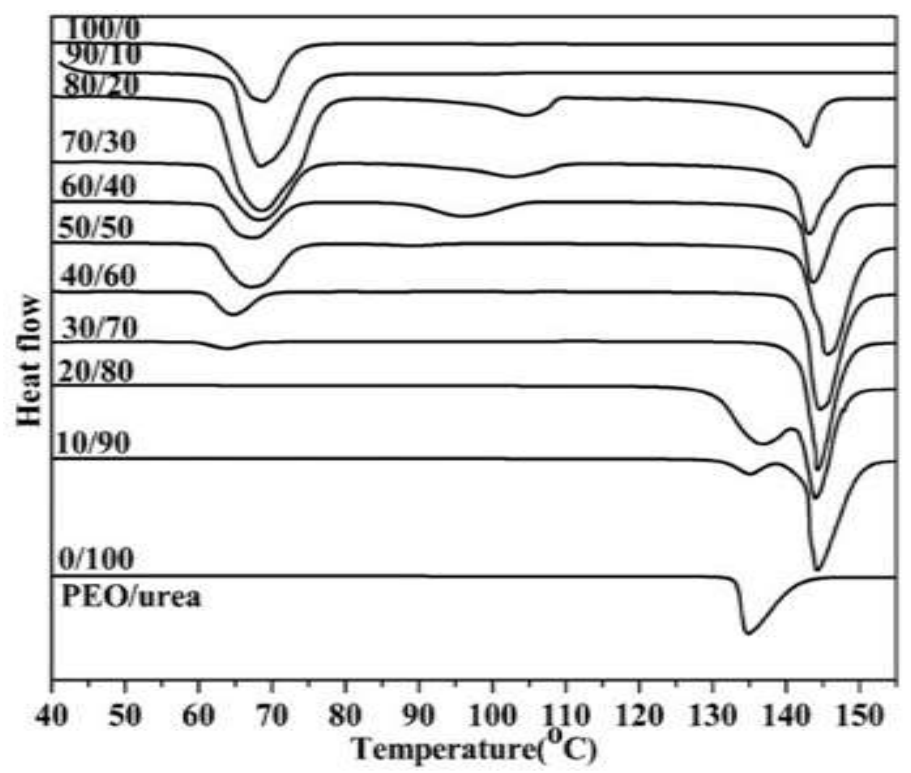

Figure 1. DSC thermograms of $\mathrm{PEO} /$ urea obtained at a heating rate of $2^{\circ} \mathrm{C} / \mathrm{min}$, showing multiple melting endotherms of neat PEO, neat urea, and their blends.

Figure 1 exhibits the DSC thermograms of various PEO/urea blends obtained at a heating rate of $2{ }^{\circ} \mathrm{C} / \mathrm{min}$. The melting transition peaks of neat PEO and neat urea are located in the vicinity of $65{ }^{\circ} \mathrm{C}$ and $135{ }^{\circ} \mathrm{C}$, respectively. It is striking to notice that some of the urea rich compositions show dual melting peaks which are comparable to or considerable higher than those of the constituents. The highest endothermic peak that is located at around $144{ }^{\circ} \mathrm{C}$ 
may be ascribed to the melting peak of the $\alpha$ crystal, formed by molecular complexation between PEO and urea molecules.

In the PEO-rich compositions, another set of exothermic peaks, albeit weak, is seen in the vicinity of $100{ }^{\circ} \mathrm{C}$, which may be attributed to the metastable $\beta$ crystal.[16, 17] According to Liu et al,[17] the $\alpha$ crystal exhibits a channel-like (cylindrical) topology, whereas the $\beta$ crystal shows a sheet-like structure. At low temperatures in the vicinity of the PEO crystal melting, the PEO rich compositions show slight change of the melting peaks (i.e., > $90 \mathrm{wt} \%$ ), but it remains virtually stationary with further decrease of PEO content.

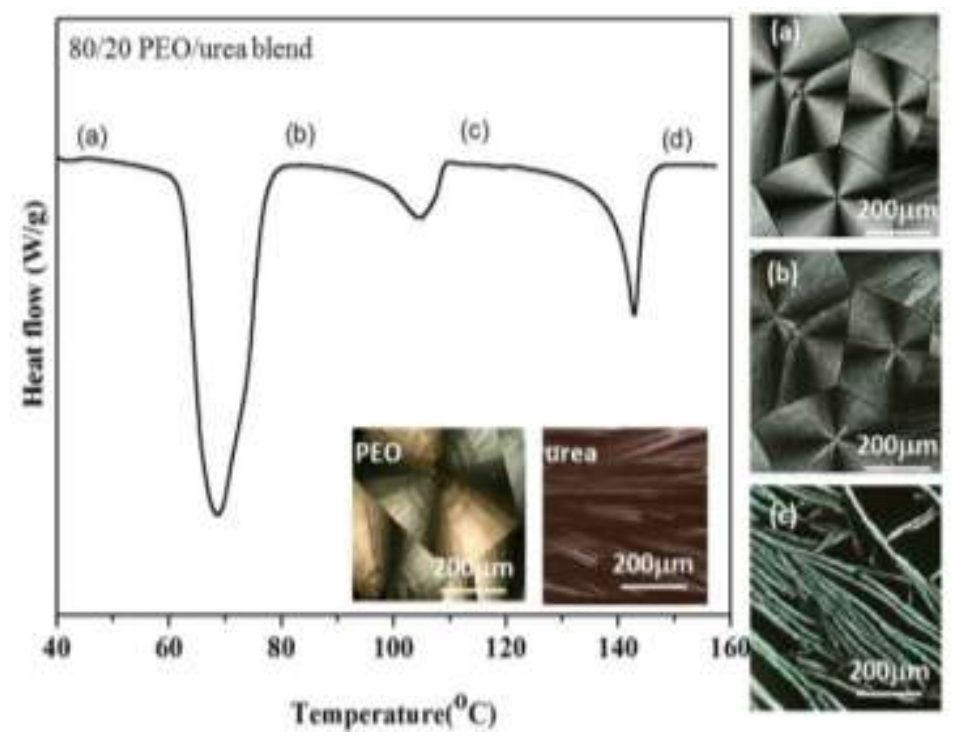

Figure 2. Polarized optical micrographs of the 80/20 PEO/urea blend, showing the hierarchical structures corresponding to the indicated temperatures of DSC thermogram: (a) spherulites of mixed $\mathrm{PEO} / \beta$ crystals at $45^{\circ} \mathrm{C}$; (b) change of spherulitic texture (sheet-like lamellae) at the core at $80^{\circ} \mathrm{C}$ and (c) needle-like cylindrical lamellae of the $\alpha$ form at $105^{\circ} \mathrm{C}$ and (d) totally dark appearance under crossed polarizers (picture not shown).

In Figure 2 are shown the DSC thermogram of the 80/20 PEO/urea composition in conjunction with the polarized optical micrographs (POM) pictures of the neat PEO and urea crystals in the inset along with those of their 80/20 mixtures at the indicated temperatures (a-d). Neat PEO exhibits a typical spherulitic morphology, whereas neat urea shows the dendritic crystals (see inset pictures). To elucidate the phase transformation of the complexed $\alpha$ and $\beta$ crystalline phases, POM micrographs of the 80/20 PEO/urea composition were taken during the course of ascending order of temperature (Figure 2 a-c). The crystalline morphology of the 80/20 PEO/urea mixture at point (a) on the DSC thermogram is a typical spherulite. Given the similarity of spherulites of neat PEO and that at point (a), which is below the PEO crystal melting temperature, PEO spherulite appears to prevail in the 80/20 PEO/urea mixture, although it is difficult to unambiguously assign to either the neat PEO crystal or the metastable $\beta$ crystal or the combination of both crystals based on the POM micrographs. 

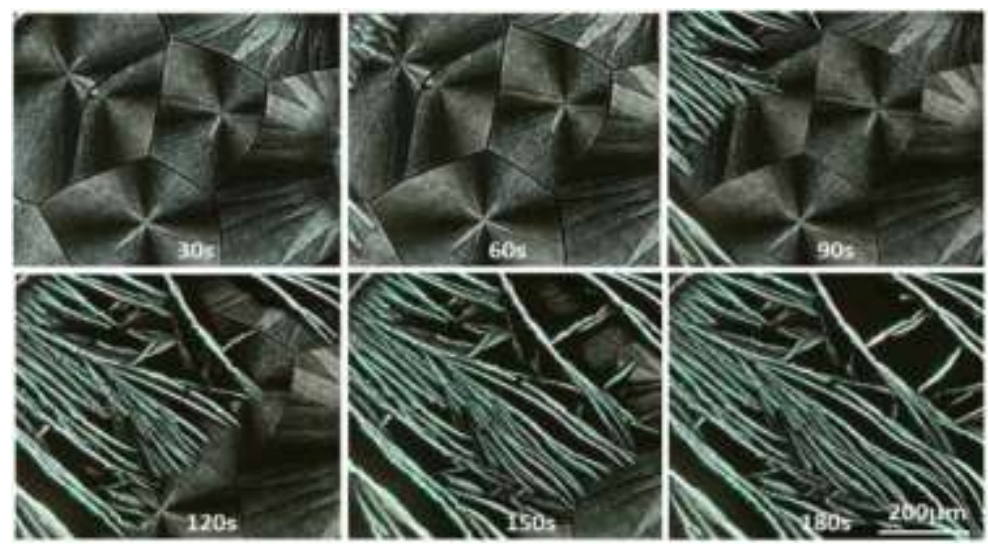

Figure 3. Polarized optical micrographs of the 80/20 PEO/urea blend taken as a function of elapsed time, showing the phase transformation from the $\beta$ (top left at 30s) to $\alpha$ (bottom right at 180s) crystal through the sequence of $\alpha+\beta$ coexistence phases. Note that the sample was kept isothermally at $105^{\circ} \mathrm{C}$.

When temperature is raised to $80^{\circ} \mathrm{C}$, the PEO crystal melts away, leaving the $\beta$ spherulitic crystal behind as manifested by noticeable change in the texture as well as contrast (see the POM picture at point (b) in Figure 2). Although the inter-spherulitic grain boundaries remain intact, one can notice the appearance of sheet-like texture spanning out from the spherulitic core as well as from the periphery boundary. However, with continued heating above the second melting peak (i.e., point (c) at $105^{\circ} \mathrm{C}$ ), the texture of $\beta$ spherulites gradually disappears while the cylindrical needle-like lamellar crystals continue to grow while showing tip splitting and branching (see Figure 3 at $30-60 \mathrm{~s}$ ) over the existing spherulites (see Figure 3 at $90-150 \mathrm{~s}$ ). The spherulitic structure eventually disappears at 180s leaving only the cylindrical lamellae behind, suggestive of phase transformation from the metastable $\beta$ phase to the stable $\alpha$ form.[18] This $\beta$ - $\alpha$ phase transformation may be regarded as the solid-solid phase transition that traverses through the narrow $\alpha+\beta$ crystal coexistence region, as typical for a first order phase transition. The cylindrical lamellar crystals finally melted away around $144^{\circ} \mathrm{C}$ corresponding to point (d) on the DSC thermograms in Figure 2, showing dark appearance (picture not included). 


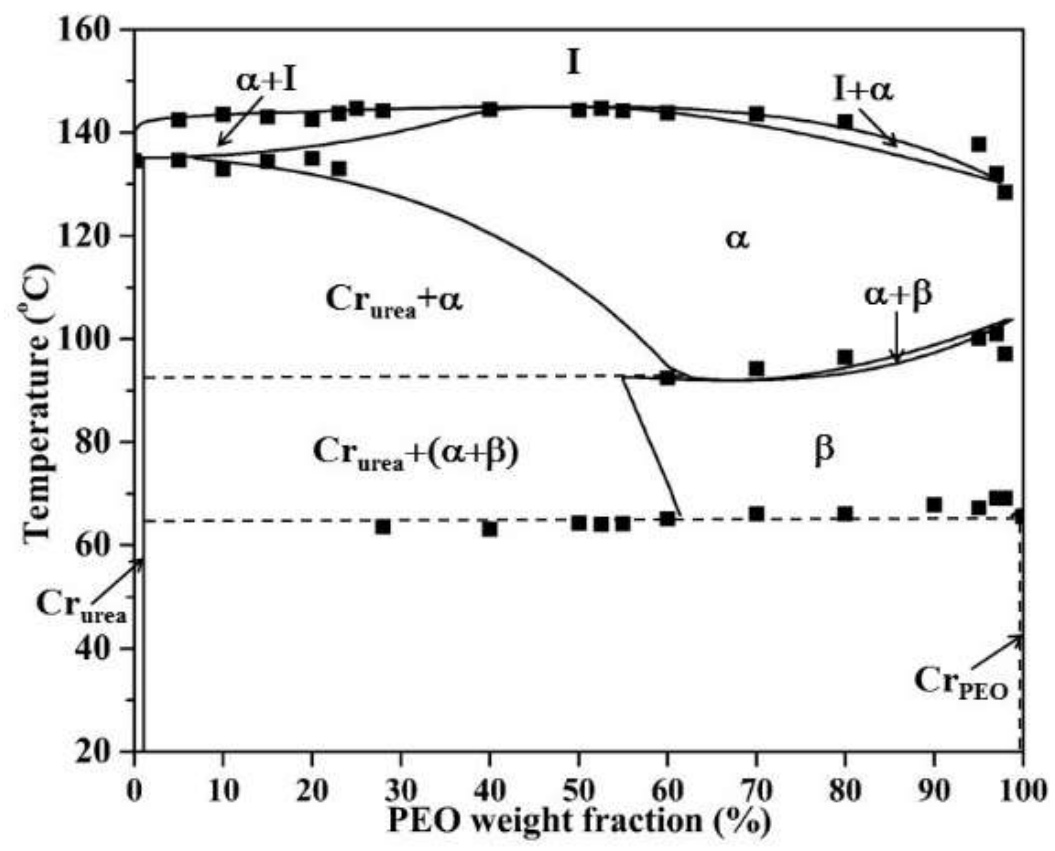

Figure 4. Self-consistently calculated phase diagram in comparison with the DSC data showing azeotrope, eutectoid with various coexistence crystal phases including the molecularly complexed $\alpha$ and/or $\beta$ crystals.

Based on these DSC melting transitions, the upper part of the phase diagram of PEO/urea was first calculated selfconsistently using the experimentally observed parameters from DSC (i.e., $\Delta \mathrm{H}_{\text {urea }}=5,000 \mathrm{~J} / \mathrm{mol}, \Delta \mathrm{H}_{\alpha}=6,000 \mathrm{~J} / \mathrm{mol}$, $\left.\mathrm{T}_{\mathrm{m}, \text { urea }}=135^{\circ} \mathrm{C}, \mathrm{T}_{\mathrm{m}, \alpha}=138^{\circ} \mathrm{C}\right)$ along with adjustable parameters $\left(\mathrm{r}_{1}=1, \mathrm{r}_{2}=40, \mathrm{~T}_{\text {crit }}=-120^{\circ} \mathrm{C}, \mathrm{A}=0\right.$ and $\left.\mathrm{c}_{\omega}=1.056\right)$. Note that $\mathrm{c}_{\omega}$ can be determined directly from the azeotropic point; otherwise it may be used as an adjustable parameter. The parameters $r_{1}$ and $r_{2}$ representing the numbers of statistical segments were estimated in proportion to the weightaverage molecular weights of each constituent. As depicted in Figure 4, the phase diagram thus obtained is an azeotrope type at elevated temperatures, which may be explained as follows. The neat urea molecules can undergo self-hydrogen bonding (i.e., intra-species) and crystallize to a network of channel-like structure. When the longchain PEO is added, the PEO chains enter into the "channels" and form the inclusion compound.[17] With the strong interspecies hydrogen bonding interaction between PEO and urea molecules, the complexed $\alpha$ crystal is thermally more stable relative to neat PEO and neat urea crystals, leading to the formation of azeotrope, showing the $\alpha$ crystal + isotropic liquid coexistence regions.

The intermediate temperature part of the phase diagram may be compared with our theoretical calculated solidus lines showing eutectoid character as depicted in Figure 4. This eutectoid reaction was captured in the present calculation using the DSC data $\left(\Delta \mathrm{H}_{\alpha}=6,000 \mathrm{~J} / \mathrm{mol}, \Delta \mathrm{H}_{\beta}=6,500 \mathrm{~J} / \mathrm{mol}\right)$ with adjustable parameters $\left(\mathrm{r}_{1}=20, \mathrm{r}_{2}=10\right.$, $\mathrm{T}_{\text {crit }}=-100^{\circ} \mathrm{C}, \mathrm{A}=0$ and $\mathrm{c}_{\omega}=0.86$ for destabilized separate crystals). Above the PEO crystal melting temperatures at higher PEO concentrations, the phase transition occurs from the stable $\alpha$ crystal (cylindrical or needle-like texture) to the $\beta$ metastable sheet-like crystal, through the $\alpha+\beta$ coexistence crystalline region with descending order of temperature (Figure 4). As the complexed $\alpha$ crystal undergoes a eutectoid reaction, it further decomposes into two 
crystal coexistence regions (urea crystal $+\alpha$ crystal and $\alpha$ crystal $+\beta$ crystal). Note that the phenomenon of the isotropic liquid of a binary crystal mixture separating into two coexistence liquid + crystalline regions accompanied by the crystalline + crystalline region is called eutectic, whereas it is called eutectoid, when one complexed solid crystal separates into two crystalline + crystalline coexistence phases.

When the temperature is further reduced below the $T_{m}$ of neat PEO, it is extremely difficult, if not impossible, to prepare stable mixed crystalline phases experimentally by solution casting. Thus the complexed crystal coexistence phase $(\alpha+\beta+$ PEO) was often described as a combined $(\alpha+\beta)$ phase in literature.[16] Given the limitation of our theory in predicting the submersed phases, it may be best to further investigate these coexistence phases experimentally on solvent cast as well as thermally annealed blends using a combination of WAXD and FTIR spectroscopic techniques.

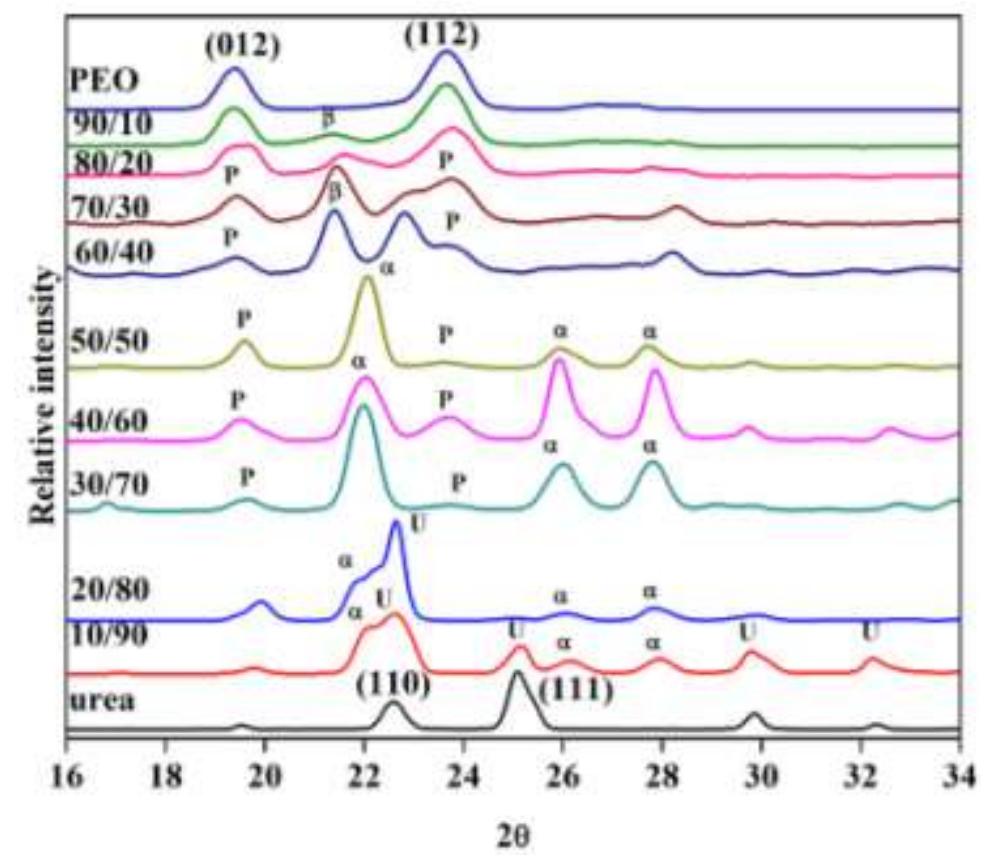

Figure 5. Bragg's scans of various PEO/urea compositions of the solvent cast blends, showing various crystalline coexistence peaks. Symbols U and P denote the urea crystal and PEO crystal for simplicity, respectively.

As depicted in Figure 5, Bragg's scans were acquired on the PEO/urea annealed blend at room temperature as a function of blend ratios to determine the crystalline coexistence phases. Considering the potential non-equilibrium nature of the solvent cast samples, thermal annealing was performed on all blends in the isotropic melt state at 150 ${ }^{\circ} \mathrm{C}$ for $10 \mathrm{~min}$. The solution cast samples and thermally annealed samples exhibited similar WAXD peaks, and thus only the Bragg's scans of the solvent cast suggesting were shown. Since there is no further change in the crystalline structure during annealing and thus it is fair to assume that these annealed mixtures have reached thermal equilibrium. Several WAXD characteristic peaks were observed at $2 \theta=22.6^{\circ}, 25.1^{\circ}$ and $29.9^{\circ}$ attributable to the neat urea crystal (see the bottom Bragg's scan) having the tetragonal lattice,[11, 25] whereas PEO shows two strong 
peaks at $2 \theta=19.4^{\circ}$ and $23.7^{\circ}$ (top WAXD scan) belonging to the monoclinic unit cell. The intermediate blends shows several new diffraction peaks, which are absent in the neat PEO or neat urea crystals, indicating the formation of new crystalline phases in these PEO/urea mixtures.

At low PEO concentrations, the tetragonal crystal developed, with several major peaks at $2 \theta=22.0^{\circ}, 25.9^{\circ}$ and $27.9^{\circ}$, which have been assigned to $\alpha$ crystal. Therefore, the observed diffraction peaks at the low PEO concentration (i.e., 10/90 PEO/urea) confirms the coexistence urea crystal $+\alpha$ crystal region within the phase diagram. On the other hand, at a very high concentration of PEO such as 80/20 PEO/urea composition, the peak at $2 \theta=21.4^{\circ}$ can be ascribed to the complexed $\beta$ crystal. For the blend compositions rich in PEO, the system can be considered as a mixture of PEO $+\beta$ crystal, whereas the WAXD scans of $40 / 60 \mathrm{PEO} /$ urea are primarily contributed by a combination of $\alpha$ crystal and PEO crystal. When PEO concentration exceeds $60 \mathrm{wt} \%$, the PEO and urea mixtures form $\beta$ crystals. The present WAXD study confirmed the existence of $\beta$ crystal in this PEO/urea system and thus it is consistent with the report of Liu and Pellerin.[16] The lack of $\beta$ crystal in the report by Wagner et al[19] may be due to the fact that these authors switched the PEO with low molar mass polyethylene glycol (PEG, $\mathrm{M}_{\mathrm{w}}=400$ ) at higher PEG concentrations, without realizing the fact that the molecular weight and the end groups could affect the complexed crystal formation and therefore the phase diagram, although the chemical structures may be similar.

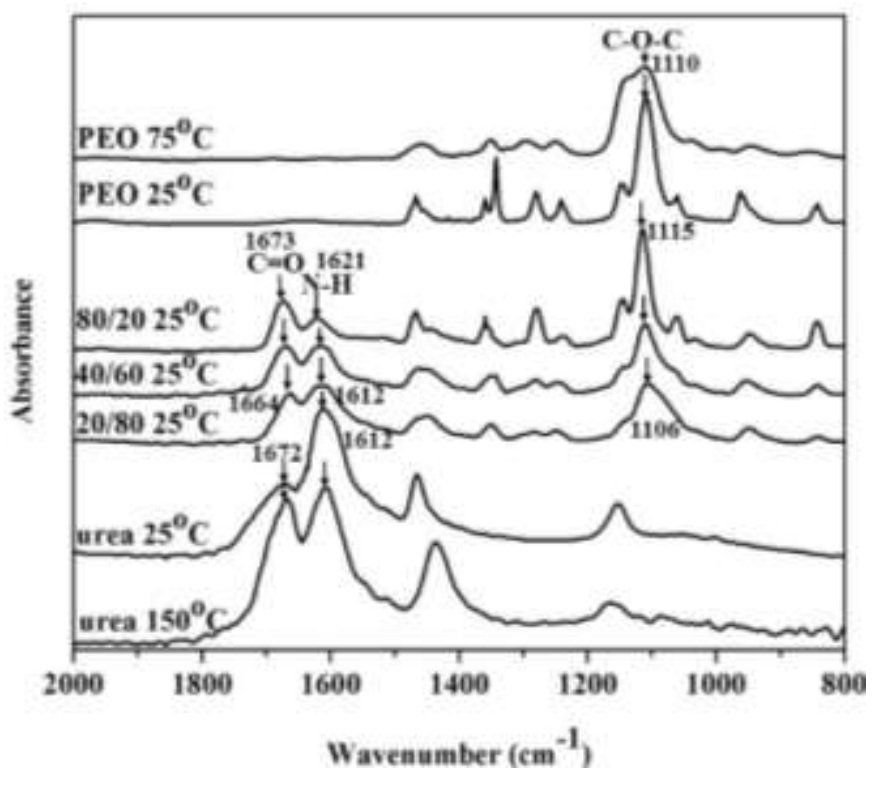

Figure 6. FTIR spectra of neat PEO, neat urea, and PEO/urea blends at various compositions, in comparison with those of neat PEO and neat urea, showing the effect of crystal melting on the IR spectra.

Figure 6 exhibits a series of FTIR spectra obtained for various PEO/urea compositions along with those of neat urea and neat PEO. The $1110 \mathrm{~cm}^{-1}$ band at $25^{\circ} \mathrm{C}$ attributable to C-O-C stretching of the neat PEO is sharp, suggesting the crystalline nature of the PEO crystal. When the temperature is increased above the melting temperature of PEO to $75^{\circ} \mathrm{C}$, the $\mathrm{C}-\mathrm{O}-\mathrm{C}$ stretching bands at $1110 \mathrm{~cm}^{-1}$ broaden, representing the amorphous (or melt) character. Upon blending at low PEO concentrations, the $\mathrm{C}-\mathrm{O}-\mathrm{C}$ stretching band moves initially to a higher wavenumber $\left(1115 \mathrm{~cm}^{-1}\right)$ 
as the $\beta$ crystal emerges and co-exists with the PEO crystal. With increasing PEO, it shifts to a lower wavenumber to $1106 \mathrm{~cm}^{-1}$, suggesting the occurrence of strong interspecies hydrogen bonding between ether oxygen of PEO chains and amine group of urea that forms the $\alpha$ crystal. The formation of interspecies hydrogen bonding interaction can be witnessed from the systematic shifts of $\mathrm{C}=\mathrm{O}$ stretching (at $1673 \mathrm{~cm}^{-1}$ ) and $\mathrm{N}-\mathrm{H}$ bending (at $1615 \mathrm{~cm}^{-1}$ ) peaks to the lower wavenumbers. Moreover, with increasing PEO content, the spectra revealed the increase in the peak height (or area) of the complexed $\beta$ crystal band at $1673 \mathrm{~cm}^{-1}$ coupled to the concomitant decrease of the band height of complexed $\alpha$ crystal at $1621 \mathrm{~cm}^{-1}$.
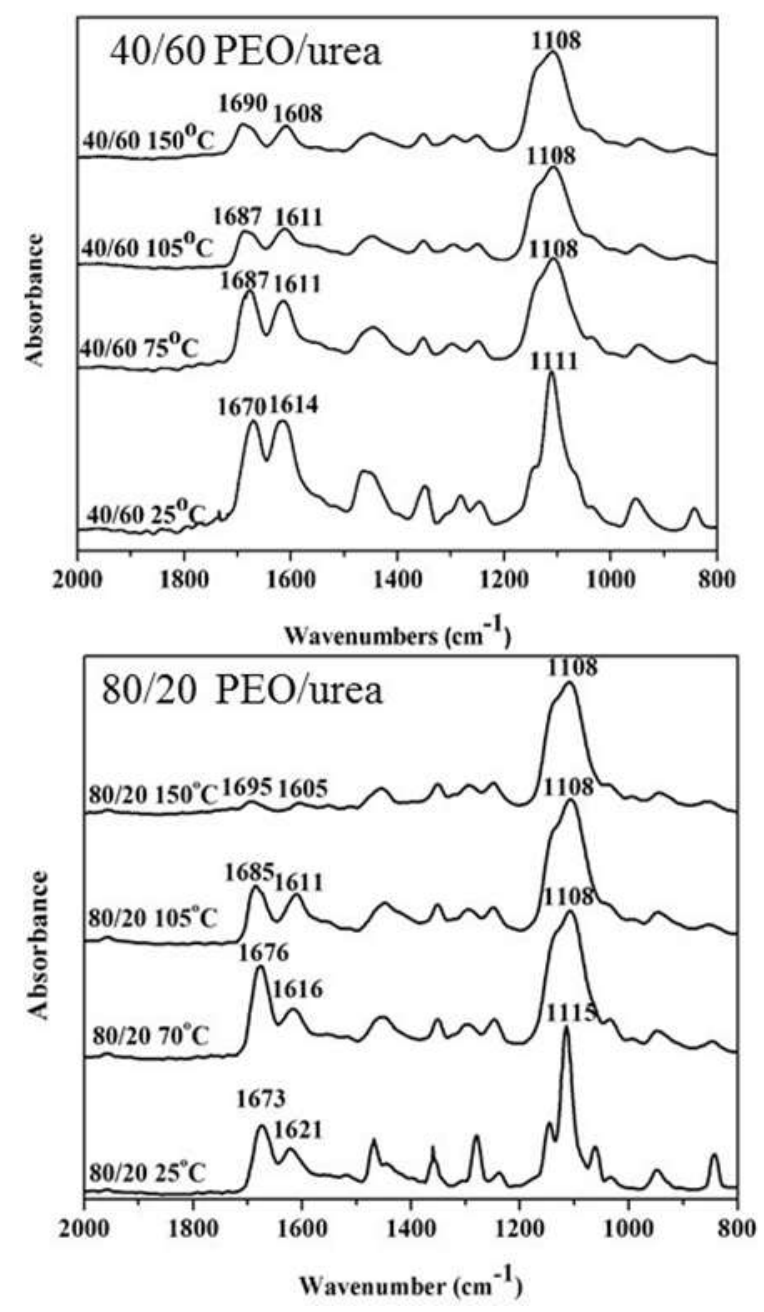

Figure 7. Variation of FTIR spectra of $40 / 60$ and $80 / 20$ PEO/urea mixtures as a function of increasing temperature, showing the melting of PEO crystal (at $75^{\circ} \mathrm{C}$ ), the $\beta-\alpha$ transition (at $105^{\circ} \mathrm{C}$ ), and the melting of $\alpha$ crystal (at $150{ }^{\circ} \mathrm{C}$ ).

The temperature dependency of FTIR spectra was further examined at various PEO/urea mixtures. Figure 7 exhibits the IR spectra of various PEO/urea samples as a function of temperatures. In the 40/60 and 80/20 PEO/urea blends, when the temperature is above the $\mathrm{T}_{\mathrm{m}}$ of PEO $\left(>65^{\circ} \mathrm{C}\right)$, the PEO bands become boarder while shifting to a lower wavenumber as a result of PEO crystal melting. In the case of $40 / 60$ blend, the $\mathrm{C}=\mathrm{O}$ stretching (at $1670 \mathrm{~cm}^{-1}$ ) shifts 
to $1687 \mathrm{~cm}^{-1}$, while the $\mathrm{N}-\mathrm{H}$ bending vibration moves from $1614 \mathrm{~cm}^{-1}$ to $1611 \mathrm{~cm}^{-1}$ during PEO crystal melting leaving the $\alpha$ crystal behind. However, there is little or no movement of both bands in the temperature range from $75^{\circ} \mathrm{C}$ to $105^{\circ} \mathrm{C}$, suggesting the lack of $\beta$ crystal phase. The $\alpha$ crystal persists during that temperature range, which eventually melts away above the $\mathrm{T}_{\mathrm{m}}\left(\right.$ at $150^{\circ} \mathrm{C}$ ) as manifested by further shifts of these $\mathrm{C}=\mathrm{O}$ and $\mathrm{N}-\mathrm{H}$ bands to 1690 and $1608 \mathrm{~cm}^{-1}$, respectively.

In the latter $80 / 20$ blend case, the $\beta-\alpha$ transition can be discerned. In the temperature range between $70^{\circ} \mathrm{C}$ and $105^{\circ} \mathrm{C}$, the $\mathrm{C}=\mathrm{O}$ stretching located at $1676 \mathrm{~cm}^{-1}$ may be assigned to the $\beta$ crystal that moves to a higher wavenumber of $1685 \mathrm{~cm}^{-1}$ upon melting to the $\alpha$ crystal phase, while the $\mathrm{N}-\mathrm{H}$ bending vibration (at $1615 \mathrm{~cm}^{-1}$ attributable to the $\beta$ crystal) shifts to a lower wavenumber of $1611 \mathrm{~cm}^{-1}$ belonging to the $\alpha$ crystal. These spectral shifts may be attributed to the phase transformation of the $\beta$ crystal to the $\alpha$ crystal. The $\beta$ crystal is composed of the PEO chains and urea networks that developed into separate stacked layers and therefore, the interaction between the PEO layer and urea layer is weak, as it is dominated by weak van der Waals force. However, in the complexed $\alpha$ crystal, the ether oxygen of PEO undergoes cross-interspecies hydrogen bonding with the $\mathrm{N}-\mathrm{H}$ of urea as the PEO chains entered the urea crystal channels. This inter-species hydrogen bonding is considerably stronger and more stable relative to that in the $\beta$ sheet crystal. Consequently, the shifting of $\mathrm{C}=\mathrm{O}$ band to a higher wavenumber and $\mathrm{N}-\mathrm{H}$ peak to a lower wavenumber above $100{ }^{\circ} \mathrm{C}$ may be attributed to interspecies hydrogen bonding between the $\mathrm{N}-\mathrm{H}$ of urea and ether oxygen of PEO chains, suggesting the freeing up of the $\mathrm{C}=\mathrm{O}$ of urea. That is to say, the original intramolecular hydrogen bonding between $\mathrm{N}-\mathrm{H}$ and $\mathrm{C}=\mathrm{O}$ of urea molecule is weaken and thus the $\mathrm{C}=\mathrm{O}$ stretching band moves to a higher wavenumber. The relative IR absorbance heights support the occurrence of this $\beta-\alpha$ phase transition. When temperature increases from $25^{\circ} \mathrm{C}$ to $105^{\circ} \mathrm{C}$, the $\beta$ crystal peak at $1670 \mathrm{~cm}^{-1}$ is suppressed, while the $\alpha$ crystal peak at $1620 \mathrm{~cm}^{-1}$ increases in magnitude.

The combined experimental evidences from DSC, WAXD and FTIR indicated the occurrence of induced $\alpha$ and $\beta$ crystals formed by molecular complexation between urea and PEO constituents. Of particular interest is that the phase transition between these two complexes occurs with temperature as manifested in the DSC and POM experiments (see Figures 2 and 3). Taking into consideration the difference in stoichiometric ratios between these two complexed $\alpha$ and $\beta$ crystals, the overall phase diagram may be drawn for the present PEO-urea binary system (Figure 8).

As shown in Figure 8, the solid lines were obtained by self-consistently solving the total free energy of combined FH-PF theory, whereas the vertical dash-dot line was drawn to illustrate various complexed crystalline coexistence regions based on the WAXD and FTIR experiments. At the low mass fractions of PEO less than $25 \mathrm{wt} \%$, the coexistence region (from ambient to $\mathrm{T}_{\mathrm{m}}$ of urea) is primarily consisted of phase separated urea crystal $\left(\mathrm{Cr}_{\text {urea }}\right)$ and the complexed $\alpha$ crystal. As explained earlier, the urea/PEO mixture exhibited azeotrope character consisted of isotropic liquid (I) and the complexed $\alpha$ crystal at elevated temperatures above $120^{\circ} \mathrm{C}$. Upon decreasing temperature, the eutectoid behavior was observed between $135-95^{\circ} \mathrm{C}$, wherein the stable $\alpha$ crystal was segregated into broad $\mathrm{Cr}_{\text {urea }}+\alpha$ crystal region and a very narrow $\alpha+\beta$ coexistence region. With further decrease temperature below $95^{\circ} \mathrm{C}$ but above $\mathrm{T}_{\mathrm{m}}$ of PEO crystals $\left(65^{\circ} \mathrm{C}\right)$, the $\alpha+\beta$ region was found to be bound in between the $\mathrm{Cr}_{\text {urea }}+\alpha$ and the pure $\beta$ phase crystal. 


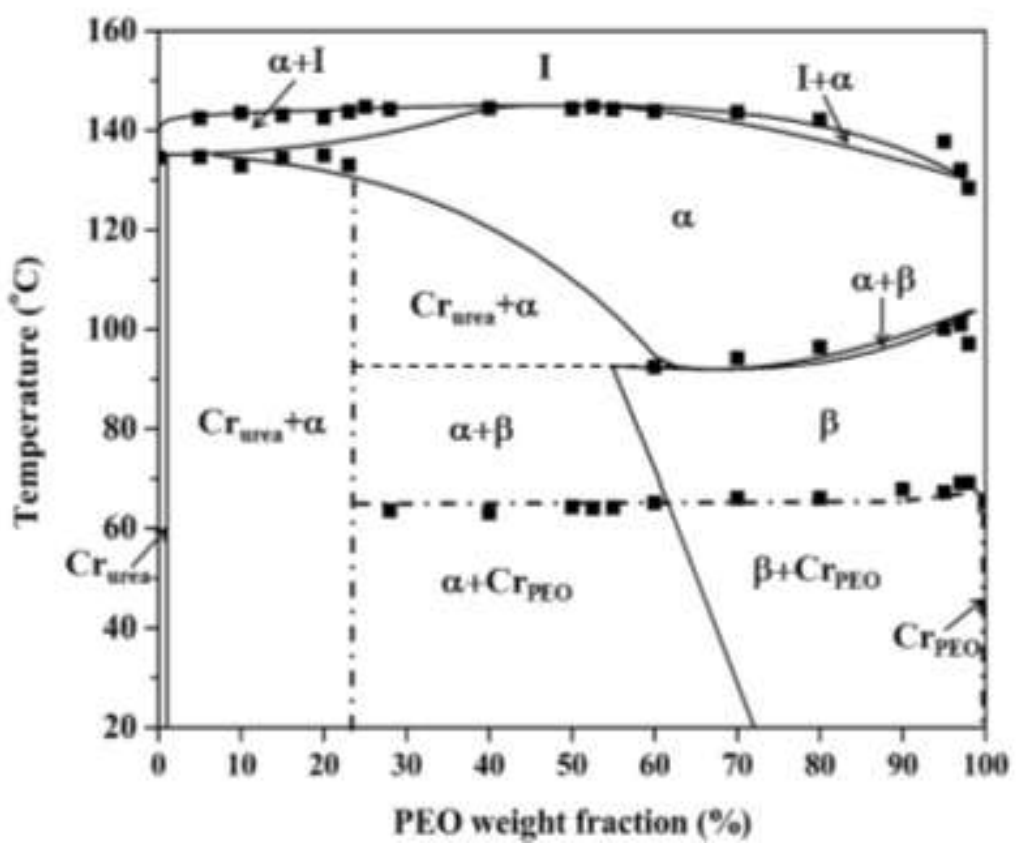

Figure 8. Overall phase diagram of PEO/urea blends in comparison with the melting transition temperatures obtained by DSC in conjunction with the demarcation lines for the complexed $\alpha$ and $\beta$ crystals. The solid line and dash line were obtained by self-consistently solving the combined Flory-Huggins free energy for liquid-liquid demixing and phase field free energy for crystal solidification. The vertical dash-dot line was drawn by hand to guide eye.

Below $\mathrm{T}_{\mathrm{m}}$ of PEO crystal, the $\mathrm{Cr}_{\text {urea }}+\alpha$ crystal region is observed at low PEO content below 23 wt $\%$ as manifested by DSC and WAXD. With increasing PEO content above $25 \mathrm{wt} \%, \mathrm{Cr}_{\text {urea }}$ is no longer discernable in the WAXD experiments. Instead, PEO crystal $\left(\mathrm{Cr}_{\mathrm{PEO}}\right)$ was found to co-exist with $\alpha$ crystal in the intermediate region of 23-55 $\mathrm{wt} \%$ and with $\beta$ crystal above $70 \mathrm{wt} \%$ of PEO (at $25^{\circ} \mathrm{C}$ ). It may be hypothesized that PEO, being a semicrystalline polymer, consists of crystalline and non-crystalline amorphous chains. Hence, the non-crystalline amorphous PEO chains can undergo molecular complexation with urea molecules leading to the formation of either $\alpha$ or $\beta$ crystal in a manner dependent on PEO concentration.

At room temperature above the mass fraction of $70 \mathrm{wt} \% \mathrm{PEO}$, this region may be characterized by the $\beta+\mathrm{Cr}_{\mathrm{PEO}}$ coexistence region. Note that the self-consistently calculated coexistence line representing the mass ratio of urea/PEO of the $\beta$ phase crystal varies from 55 to $72 \mathrm{wt} \%$ depending on temperature, which is, in average, analogous to the 2:3 molar ratio.[16] However in the present case, the urea/PEO ratio in the complexed $\beta$ crystal phase may be composition and temperature dependent in contrast to the fixed 2:3 ratio. The present assignment of $\alpha+\mathrm{Cr}_{\mathrm{PEO}}$ in the intermediate region and $\beta+\mathrm{Cr}_{\mathrm{PEO}}$ coexistence crystal region in the high $\mathrm{PEO}$ concentration is primarily due to the fact that the complexed $\alpha$ crystal undergoes phase transformation to the $\beta$ crystal with the increase of PEO content. At the extreme PEO concentration (e.g., > 99 wt \%), the PEO crystal region may exist like a pure form as manifested by the transition line that is coincided with the pure PEO axis. 


\section{Conclusions}

The phase diagram of PEO/urea mixtures was investigated both experimentally and theoretically. The observed solid-liquid phase diagram of PEO/urea blend was explained in the context of the combined Flory-Huggins free energy for liquid-liquid demixing and phase field free energy of crystal solidification. The phase diagram of PEO and urea mixture was found to be of azeotrope type consisting of urea crystal and the complexed $\alpha$ crystal. With decreasing temperature, the eutectoid behavior was observed, wherein the stable $\alpha$ crystal was segregated into the urea crystal $+\alpha$ crystal and $\alpha$ crystal $+\beta$ crystal coexistence regions. Below the melting temperature of PEO, three regions can be identified; urea crystal $+\alpha$ crystal, $\alpha$ crystal + PEO crystal and $\beta$ crystal + PEO crystal coexistence regions. The change of $\alpha$ crystal + PEO crystal to $\beta$ crystal + PEO crystal coexistence regions with increasing PEO concentration suggests that the $\alpha$ crystal transform to the $\beta$ crystal with increasing PEO content above 60 wt $\%$. To the best of the authors' knowledge, the present paper is the first to identify the azeotrope and eutectoid behavior in the phase diagram of this PEO/urea system.

Note

We do not have conflict of interest to declare.

\section{Acknowledgements}

Support of this work by the National Science Foundation, Division of Materials Research, Polymer Program, NSF-DMR 1161070 is greatly acknowledged. This paper is dedicated to our dear friend and colleague, Dr. Tisato Kajiyama for his outstanding contribution in polymer physics and envious career as President of Kyushu University.

\section{References}

[1] G. Allegra, M. Farina, A. Immirzi, A. Colombo, U. Rossi, R. Broggi, G. Natta, Inclusion compounds in perhydrotriphenylene. Part I. The crystal structure of perhydrotriphenylene and of some inclusion compounds, J. Chem. Soc. B (1967) 1020-1028.

[2] M. Farina, G. Natta, G. Allegua, M. Löffelholz, Inclusion compounds of linear polymers and polymerization of monomers included in perhydrotriphenylene, J. Poly. Sci. C 16(5) (1967) 2517-2524.

[3] M. Farina, U. Pedretti, M.T. Gramegna, G. Audisio, Stereospecific and Asymmetric Inclusion Polymerization I. Polymerization of trans-and cis-1, 3-Pentadiene Included in Racemic Perhydrotriphenylene, Macromolecules 3(5) (1970) 475-480.

[4] A. Colombo, G. Allegra, Single Crystal to Single Crystal Transformation of Perhydrotriphenylene Inclusion Compounds during Canal Polymerization of Butadiene, Macromolecules 4(5) (1971) 579-584.

[5] K.D. Harris, P. Jonsen, 2 H NMR investigation of the dynamic behaviour of n-hexadecane in its urea inclusion compound, Chem. Phys. Lett. 154(6) (1989) 593-598.

[6] D. Kenneth, Structural aspects of urea inclusion compounds and their investigation by X-ray diffraction: a general discussion, J. Chem. Soc., Faraday Trans. 86(17) (1990) 2985-2996.

[7] K.D. Harris, Investigating the structure and dynamics of a family of organic solids: the alkane/urea inclusion compounds, J. Solid State Chem. 106(1) (1993) 83-98.

[8] A.E. Aliev, K.D. Harris, Conformational properties of monosubstituted cyclohexanes in their thiourea inclusion compounds and in solution: variable-temperature one-dimensional and two-dimensional carbon-13 NMR investigations, J. Am. Chem. Soc. 115(14) (1993) 6369-6377.

[9] C. Choi, D. Davis, A. Tonelli, Inclusion compound formed between poly (. epsilon.-caprolactone) and urea, Macromolecules 26(6) (1993) 1468-1470.

[10] A.E. Tonelli, Crystalline polymer inclusion compounds: potential models for the behaviour of polymer chains in their bulk, ordered phases, Polymer 35(3) (1994) 573-579. 
[11] N. Vasanthan, I.D. Shin, A.E. Tonelli, Structure, conformation, and motions of poly (ethylene oxide) and poly (ethylene glycol) in their urea inclusion compounds, Macromolecules 29(1) (1996) 263-267.

[12] K. Suehiro, Y. Nagano, Structural studies on molecular complexes of polyethers, 1. Urea - ethylene oxide oligomer complexes, Die Makromolekulare Chemie 184(3) (1983) 669-674.

[13] A. Chenite, F. Brisse, Structure and conformation of poly (ethylene oxide), PEO, in the trigonal form of the PEO-urea complex at $173 \mathrm{~K}$, Macromolecules 24(9) (1991) 2221-2225.

[14] B. Bogdanov, M. Michailov, C. Uzov, G. Gavrailova, Phase transformation on heating of undercooled melt of poly (ethylene oxide) - urea molecular complex, Macromol. Chem. Phys. 195(6) (1994) 2227-2231.

[15] B. Bogdanov, M. Michailov, C. Uzov, G. Gavrailova, Complexation of poly (ethylene oxide) and urea, J. Polym. Sci. B: Polym. Phys. 32(2) (1994) 387-394.

[16] Y. Liu, H. Antaya, C. Pellerin, Characterization of the stable and metastable poly (ethylene oxide)-urea complexes in electrospun fibers, J. Phys. Chem. B 46(18) (2008) 1903-1913.

[17] Y. Liu, C. Pellerin, Stability and phase behavior of the poly (ethylene oxide)-urea complexes prepared by electrospinning, Polymer 50(12) (2009) 2601-2607.

[18] Y. Liu, H. Antaya, C. Pellerin, Structure and Phase Behavior of the Poly (ethylene oxide)- Thiourea Complex Prepared by Electrospinning, J. Phys. Chem. B 114(7) (2010) 2373-2378.

[19] J.F. Wagner, M. Dosiere, J.M. Guenet, Temperature - Concentration Phase Diagram of PEO - Urea, Macromol. Symp. 222(1) (2005) 121-124.

[20] M.L. Huggins, Solutions of long chain compounds, J. Chem. Phys. 9(5) (1941) 440-440.

[21] P.J. Flory, Thermodynamics of high polymer solutions, J. Chem. Phys. 10(1) (1942) 51-61.

[22] R. Kobayashi, Modeling and numerical simulations of dendritic crystal growth, Phys. D: Nonlinear Phenom. 63(3) (1993) 410-423.

[23] R.A. Matkar, T. Kyu, Phase diagrams of binary crystalline-crystalline polymer blends, J. Phys. Chem. B 110(32) (2006) 16059-16065.

[24] P. Rathi, T.-M. Huang, P. Dayal, T. Kyu, Crystalline-- Amorphous Interaction in Relation to the Phase Diagrams of Binary Polymer Blends Containing a Crystalline Constituent, J. Phys. Chem. B 112(20) (2008) 64606466.

[25] A. Smith, The Crystal Structure of Urea - Hydrocarbon and Thiourea - Hydrocarbon Complexes, J. Phys. Chem. B 18(1) (1950) 150-151. 